\title{
Conformation of the Posterior Fossa of the Skull: A Predisposing Factor in Patients with Hemifacial Spasm
}

\author{
Gyu-Sung Kang, Ha-Young Choi, Jong-Myong Lee, Eun Jeong Koh, Jung-Soo Park
}

Department of Neurosurgery, Research Institute of Clinical Medicine of Chonbuk National University, Biomedical Research Institute of Chonbuk National University Hospital, Jeonju, Republic of Korea

\author{
Corresponding author: Ha-Young Choi \\ Department of Neurosurgery, \\ Chonbuk National University Hospital, \\ Chonbuk National University, 20, \\ Geonjiro Deokjin-gu, Jeonju 54907, \\ Republic of Korea \\ Tel: $+82-63-250-1870$ \\ Fax: $+82-63-277-3273$ \\ E-mail: cbnu23450@gmail.com
}

Received: June 30, 2020

Revised: August 3, 2020

Accepted: August 12, 2020
Objective: Hemifacial spasm (HFS) can be caused by vascular compression of the facial nerve at the root entry zone in the brain stem. The study aims to analyze the length and volume of the posterior cranial fossa (PCF) and the angle of skull flatness to evaluate any possible contribution of morphological characteristics to the development of neurovascular compression leading to HFS. Methods: We enrolled 30 patients with HFS and matched them by age and sex to controls. Three-dimensional volumetric analyses of the PCF were performed using magnetic resonance imaging. We measured the angle and approximate volume of the PCF using the ellipsoid method. Results: The mean volume of the PCF in patients with HFS was significantly lower $(220.2 \pm 24.9$ vs. $243.1 \pm 31.2 ; p=0.003)$ than in controls. In addition, the mean angle of the PCF of patients with HFS was smaller than that of controls $(100.7 \pm 6.0$ vs. $106.5 \pm 7.0 ; p=0.001$ ). Several clinical factors were investigated, and there were significant associations between PCF volume and hypertension. Hypertension is more common in the HFS patient group than in the control group (18/30 [60.0\%] vs. $10 / 30$ [33.3\%]; $p=0.038$ ). Conclusion: Our results suggest that patients with HFS can be characterized by flatness of the skull and small volume of the PCF. These structural differences may play a role in neurovascular compression in patients with HFS.

Key Words: Cranial fossa, posterior; Hemifacial spasm; Skull

\section{INTRODUCTION}

Hemifacial spasm (HFS) is a neuromuscular movement disorder generally characterized by unilateral, involuntary, and intermittent contractions of the muscles innervated by the ipsilateral facial nerve (cranial nerve VII $)^{1)}$. Patients with HFS suffer from facial muscle spasms which can impact social interaction with others and their quality of life ${ }^{1)}$. The most common cause of HFS is a neurovascular conflict between the artery and the facial nerve at the root entry zone of the brainstem ${ }^{13}$. A number of mechanisms likely underly the development of neurovascular conflict leading to HFS. The incidence of HFS is increased in the presence of hypertension (HTN) in individuals older than 50 years, women, and Asians 5).

Anatomical features that characterize patients with HFS have been reported. Deviation of the vertebra-basilar arteries to the lateral position, where the facial nerve is located, was previously shown to be associated with $\mathrm{HFS}^{(6)}$. The possibility of a morphological difference in the infratentorial space of patients with HFS has been investigated because a smaller infratentorial cerebrospinal fluid spaces can increase the risk of neurovascular $\operatorname{conflict}^{10)}$

Whether a structural difference in the infratentorial space of the cranium is associated with the development of neurovascular conflict leading to HFS remains a matter of debate. We performed a morphometric study of the posterior cranial fossa (PCF) on 3-dimensional (3D) high-resolution magnetic resonance imaging (MRI) scans of patients with HFS. Our results were compared to the findings from age- and sex-matched controls to evaluate possible subtle differences in the 3D conformation of the infratentorial space in patients with HFS.

\section{MATERIALS AND METHODS}

\section{Patients}

Between March 2017 and April 2019, 41 patients with HFS underwent neuroimaging studies at our Department of Neurosurgery for evaluation of surgical indications. The patients who were not tested out MRI imaging or deficient adequate sequences of MRI were excluded. The medical records were reviewed to collect the clinical information of sex, age, and affected side. 

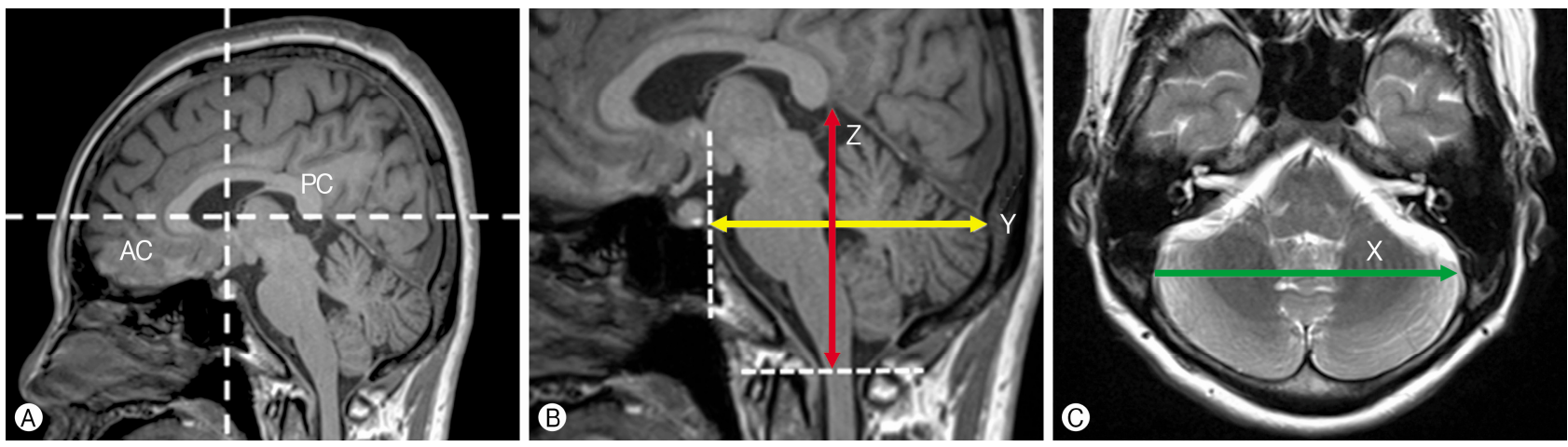

Fig. 1. Method for morphologic analysis of the posterior cranial fossa. (A) Horizontal and vertical white dotted lines indicate the $Y$ - and Z-axis on a mid-sagittal slice, respectively. The anterior commissure (AC) and posterior commissure (PC) are shown as the origin and a point on the $Y$ - and $Z$-axis is the vertical line to the $Y$-axis. (B) The length of the posterior cranial fossa ( $Y$, yellow line) was measured along the $Y$-axis from the vertical dotted line parallel to $Z$-axis through the posterior clinoid process to the sinus confluence. The height of the posterior cranial fossa ( $Z$, red line) is measured along the $Z$-axis from the uppermost point of the straight sinus to the horizontal dotted line parallel to $Y$-axis through the posterior edge of the foramen magnum. (C) A axial slice showing measurements along the $X$-axis. The width of the posterior cranial fossa ( $X$, green line) was measured along the $X$-axis between bilateral transverse-sigmoid sinus junctions.

Our final study population was comprised of $30 \mathrm{HFS}$ patients. All patients were tested for electroneuronography as preoperative evaluation, and abnormal results were confirmed in each lesion direction, such as the lateral spread response study shown stimulating facial nerve and recording from muscles. Age- and sex-matched controls were recruited from 30 patients with headache whose neuroimaging evaluation to exclude intracranial lesions returned normal findings.

\section{Imaging Analysis}

All subjects underwent a standard MRI protocol (Philips Achieva, 3.0T; Philips Health, Andover, MA, USA), including conventional axial T1-weighted image (WI), axial T2-WI, time of flight, and constructive interference in steady state image.

The morphology and volume of the PCF were evaluated by approximation to an ellipsoid in accordance with the method introduced by Hamasaki et al. ${ }^{6}$ in a study of HFS. Specifically, length, width, and height of the ellipsoid were determined on 3D high-resolution MRI (Fig. 1).

Horizontal and vertical white dotted lines indicate the Yand Z-axes on a mid-sagittal slice, respectively. The anterior commissure and posterior commissure are shown as the origin, and a point on the $\mathrm{Y}$-axis and $\mathrm{Z}$-axis is the vertical line to the $\mathrm{Y}$-axis. Length $(\mathrm{Y})$ was defined as the distance from the posterior clinoid process to the sinus confluence along the mid-sagittal plane of MRI: anteroposterior commissure line (Y-axis), height (Z) was defined as the distance from the uppermost portion of the straight sinus to the foramen magnum along the line per pendicular to the anteroposterior commissure line (Z-axis). And width (X) was defined as the distance between the bilateral transverse-sigmoid sinus junctions along the rightleft axis (Xaxis). The morphology and volume of the PCF were evaluated

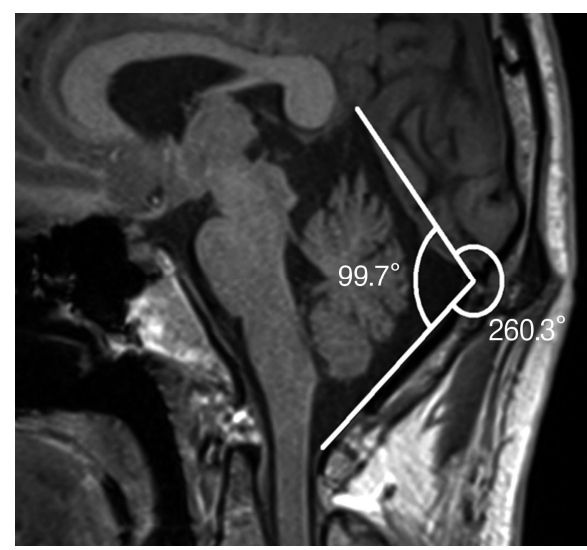

Fig. 2. The angle of skull flatness was measured using the mid-sagittal slice.

by measuring absolute $\mathrm{X}, \mathrm{Y}$, and $\mathrm{Z}$ values, the ratio of $\mathrm{Z}$ to $\mathrm{X}$ extent $(\mathrm{Z} / \mathrm{X})$, the ratio of $\mathrm{Z}$ to $\mathrm{Y}$ extent $(\mathrm{Z} / \mathrm{Y})$, and the volume $(\pi X Y Z / 6)$ of the ellipsoid ${ }^{5}$.

The angle of skull flatness was measured using the midsagittal slice. In mid-sagittal space, we measured a straight line past the tentorium cerebelli. And we made another line from the foramen magnum to the tentorium cerebelli along the point of the occipital bone inner table. We considered the angle measured between these 2 straight lines, the angle of PCF (Fig. 2).

\section{Statistical Analysis}

Differences between groups were analyzed using the student's $t$ test, $\mathrm{X}^{2}$ test, and paired $t$-test as appropriate for continuous and categorical variables. Statistically significant differences were defined as p-value less than 0.05 . 


\section{RESULTS}

\section{Baseline Characteristics}

The study included 9 men and 21 women with HFS ( $n=30)$ and their age and sex-matched controls ( 9 men and 21 women, $\mathrm{n}=30$ ); the mean age was $55.1 \pm 12.6$ (range, 36-78) and 55.9 \pm 8.2 years (range, 34-80), respectively. A significant difference was detected in the history of HTN between the patients with HFS and the control group (18/30 [60\%] vs. 10/30 [33.3\%]; $\mathrm{p}=$ 0.038) (Table 1).

\section{Radiologic Characteristics}

In the patient group, arterial contact with the facial nerve roots was demonstrated by preoperative neuroimaging studies and microvascular decompression surgery. The most prominent result of the study was that flatness of the PCF was associated with measured volume $\left(\mathrm{mm}^{3}\right)$ and angle. The mean volume of the PCF in patients with HFS and that of controls was (220.2 \pm 24.9 vs. $\left.243.1 \pm 31.2 \mathrm{~mm}^{3} ; \mathrm{p}=0.003\right)$. The mean angle of the PCF in patients with HFS and that of controls was $(100.7 \pm 6.0$ vs. 106.5 $\pm 7.0 ; \mathrm{p}=0.001)$. For the morphologic shape measurement of the 3 axes, only Height $(Z)$ was statistically significant $(64.7 \pm 3.0$ vs. $67.6 \pm 5.1 \mathrm{~mm} ; \mathrm{p}=0.010)$. There was no statistically significant difference in length $(\mathrm{Y})$ nor width $(\mathrm{X})$. The smaller $\mathrm{Z}$ values were measured in the surgical group, which resulted

Table 1. Comparison of baseline characteristics between patients with hemifacial spasm and controls

\begin{tabular}{lrrr}
\hline \hline & $\begin{array}{c}\text { Patients group } \\
(\mathrm{n}=30)\end{array}$ & $\begin{array}{c}\text { Control group } \\
(\mathrm{n}=30)\end{array}$ & $\mathrm{p}$-value \\
\hline Mean age (year) & $55.1 \pm 12.6$ & $55.9 \pm 8.2$ & 0.102 \\
Female & $21(70.0)$ & $22(73.3)$ & 0.774 \\
HTN & $18(60.0)$ & $10(33.3)$ & 0.038 \\
DM & $7(23.3)$ & $8(26.7)$ & 0.766 \\
\hline
\end{tabular}

HTN: hypertension; DM: diabetes mellitus.

Table 2. Comparison of anatomical characteristics between patients with hemifacial spasm and controls

\begin{tabular}{lccc}
\hline \hline & $\begin{array}{c}\text { Patients group } \\
(\mathrm{n}=30)\end{array}$ & $\begin{array}{c}\text { Control group } \\
(\mathrm{n}=30)\end{array}$ & -value \\
\hline$X(\mathrm{~mm})$ & $98.0 \pm 4.7$ & $99.9 \pm 4.1$ & 0.096 \\
$Z(\mathrm{~mm})$ & $64.7 \pm 3.0$ & $67.6 \pm 5.1$ & 0.010 \\
$Y(\mathrm{~mm})$ & $66.3 \pm 5.3$ & $68.7 \pm 5.2$ & 0.078 \\
Volume (cc) & $220.2 \pm 24.9$ & $243.1 \pm 31.2$ & 0.003 \\
Angle & $100.7 \pm 6.0$ & $106.5 \pm 7.0$ & 0.001 \\
$Z / X(\%)$ & $66.2 \pm 4.1$ & $67.7 \pm 5.9$ & 0.048 \\
$Z / Y(\%)$ & $98.2 \pm 8.8$ & $98.8 \pm 8.8$ & 0.815 \\
\hline
\end{tabular}

in a statistically low volume and angle (Table 2).

\section{DISCUSSION}

HFS is characterized by initially progressive, involuntary, irregular, tonic, clonic movements of muscles innervated by the facial nerve on the impacted side ${ }^{2,11)}$. It often starts in the orbicularis oculi and orbicularis oris muscles, but may spread to all muscles supplied by the affected facial nerve. The condition may have different causes, but compression of the facial nerve root exit zone by a vascular structure is considered the most common ${ }^{2,12)}$.

Furthermore, several cases of HFS have been associated with cerebellar hematoma, cerebellar gangliocytoma, Paget disease, and hydrocephalus, suggesting that narrowing of the posterior fossa caused by these abnormalities may increase the chance of vascular compression of the facial nerve ${ }^{2)}$.

The incidence of HFS is increased in individuals older than 50 years, women, and Asians ${ }^{6}$.

In a multicenter study, a significantly higher prevalence of HTN was found in patients with HFS than in patients with other neurologic diseases ${ }^{3,9,14)}$. In our study, the prevalence of HTN was significantly higher in HFS patients than controls, particularly in female patients $(n=21[70.0 \%])$.

Although epidemiologic studies of HFS performed in Asia remain scarce, most neurologists have observed that HFS seems more common among Asian patients ${ }^{6}$. Moreover, structural differences of the posterior fossa and temporal bone between Asian and Caucasian populations have been rarely reported ${ }^{7}$.

Generally, the size and shape of the skull varies in different races $^{4}$. According to previous studies, Caucasians have relatively long and narrow PCF's when compared to people of Asian or African descent. They have less pronounced cheek bones and exhibit elongated chins. Whereas Asian skulls tend to be shorter and broader when compared with Caucasian or African skulls. The cheek bones are wide, flare out to the sides of the skull and are forward-sloping. Particularly, the occipital profile is especially angular, and the nuchal muscle markings are moderate ${ }^{4,8}$.

These observations led us to hypothesize that flatness of PCF may be a predisposing factor for HFS. In this study, we designed a retrospective, case-control study to test this hypothesis by measuring and comparing the angle, volume, and length of the PCF.

We extracted the patients who had HFS within a certain period of time and set up a control group who were asymptomatic and had normal imaging results. The length was measured as the bilateral transverse-sigmoid sinus junctions along the right-left $(\mathrm{X})$, distance from the posterior clinoid process to the sinus confluence along the midsagittal plane $(\mathrm{Y})$ and the distance from the uppermost portion of the straight sinus to the foramen magnum $(\mathrm{Z})$ were measured at the midsagittal plane using an axial scan. The volume of the PCF was evaluated 
as an ellipsoid, measured as $\pi \mathrm{XYZ} / 6$. The angle of skull flatness was measured using a mid-sagittal slice.

Among the parameters measured in our study, the most significant statistical values were related to differences in the volume and angle of the PCF. The mean PCF volume in patients with HFS was 9.5\% smaller than in controls ( $\mathrm{p}=0.003)$, and the mean PCF angle in patients with HFS was 5.5\% lower than in controls $(\mathrm{p}=0.001)$.

As we mentioned, Asian skulls tend to be shorter and broader when compared with others. These morphological differences cause a tendency of having an angular occipital profile and flattened PCF. Our research suggests a correlation between the high incidence of HFS in Asians and the morphological differences in the skull.

Some limitations need to be considered in the present study. First, a relatively small number of cases were analyzed, which may limit the statistical power of our analyses. Second, our statistical findings may not support the relationship between the flatness of the PCF and the variation of cranial neurovascular structure as has been reported.

\section{CONCLUSION}

Our present results suggest that patients with HFS had a flatter skull than controls. The prevalence of HTN was significantly higher than in the control group. Furthermore, there is a greater prevalence in women than men. These data indirectly confirm the hypothesis that the flatness of the skull of Asians is a predisposing factor to HFS.

\section{CONFLICTS OF INTEREST}

No potential conflict of interest relevant to this article was reported.

\section{REFERENCES}

1. Bao F, Wang Y, Liu J, Mao C, Ma S, Guo C, et al.: Structural changes in the CNS of patients with hemifacial spasm. Neuro- science 289:56-62, 2015

2. Cheng J, Fang Y, Zhang H, Lei D, Wu W, You C, et al.: Quantitative study of posterior fossa crowdedness in hemifacial spasm. World Neurosurg 84:920-926, 2015

3. Defazio G, Berardelli A, Abbruzzese G, Coviello V, De Salvia R, Federico F, et al.: Primary hemifacial spasm and arterial hypertension: A multicenter case-control study. Neurology 54:11981200, 2000

4. Durbar US: Racial variations in different skulls. J Pharm Sci Res 6:370-372, 2014

5. Fukuoka T, Nishimura Y, Hara M, Nomura K, Ryu H, Yoshikawa S, et al.: Flat posterior cranial fossa affects outcomes of microvascular decompression for trigeminal neuralgia. World Neurosurg 111:e519-e526, 2018

6. Hamasaki T, Yamada K, Kitajima M, Kuratsu J: Flatness of the infratentorial space associated with hemifacial spasm. Acta Neurochir (Wien) 158:1405-1412, 2016

7. Jannetta PJ, Abbasy M, Maroon JC, Ramos FM, Albin MS: Etiology and definitive microsurgical treatment of hemifacial spasm. Operative techniques and results in 47 patients. J Neurosurg 47:321-328, 1977

8. Johnson DR, O'Higgins P, Moore WJ, McAndrew TJ: Determination of race and sex of the human skull by discriminant function analysis of linear and angular dimensions. Forensic Sci Int 41:41-53, 1989

9. Li F, Liu R: Clinical analysis of microvascular decompression in patients with hemifacial spasm: A retrospective study. Ann Palliat Med 9:318-323, 2020

10. Mercier P, Sindou M: The conflicting vessels in hemifacial spasm: Literature review and anatomical-surgical implications. Neurochirurgie 64:94-100, 2018

11. Nilsen B, Le KD, Dietrichs E: Prevalence of hemifacial spasm in Oslo, Norway. Neurology 63:1532-1533, 2004

12. Rosenstengel C, Matthes M, Baldauf J, Fleck S, Schroeder H: Hemifacial spasm: Conservative and surgical treatment options. Dtsch Arztebl Int 109:667-673, 2012

13. Rudzińska M, Wójcik-Pędziwiatr M, Malec M, Grabska N, Hartel M, Szczudlik A: Small volume of the posterior cranial fossa and arterial hypertension are risk factors of hemifacial spasm. Neurol Neurochir Pol 48:383-386, 2014

14. Zhong J, Li ST, Zhu J, Guan HX, Zhou QM, Jiao W, et al.: A clinical analysis on microvascular decompression surgery in a series of 3,000 cases. Clin Neurol Neurosurg 114:846-851, 2012 